\title{
Comparative study of efficacy and safety of Iron polymaltose complex with ferrous sulphate in antenatal women with moderate anemia
}

\author{
Chandrakala Kambar ${ }^{1}$, Zahedabano $^{2}$, Meenakumari $\mathrm{A}^{3}$ \\ ${ }^{1}$ Associate Professor of Pharmacology, Siddhartha Medical college, Vijayawada \\ ${ }^{2}$ Professor and Head, Department of Pharmacology, Guntur Medical College, Guntur. \\ ${ }^{3}$ Professor, Department of Pharmacology, Guntur Medical College, Guntur
}

\begin{abstract}
Iron deficiency is the most common cause of anemia in pregnancy. The conventional iron preparations with ferrous salts showed noncompliance due to various side effects when compared to iron polymaltose complex. But the efficacy of iron polymaltose complex (IPC) in the treatment of iron deficiency anaemia (IDA) during pregnancy has not been established, and the evidence is inconclusive. The aim of this study was to compare efficacy, safety, compliance and cost-effectiveness of ferrous sulphate (FS) with IPC in antenatal women. 100 antenatal women with 14 to 20 weeks of gestation were recruited in the study, one group of 50 women were given FS and the other group of 50 women were given IPC for 6 weeks. Haemoglobin concentration (Hb), Packed cell volume(PCV), Mean Corposcular Haemoglobin concentration and serum iron were estimated before and after treatment with the above iron formulations. Compliance with pill count and safety with adverse effects monitoring in followup were noted. Statistical analysis was done with student's T test and chi square test. The improvement of haematological parameters was comparable in both groups statistically but compliance and safety were better with the IPC group when compared to FS group. IPC is a better alternative to FS as it is safe and showed more compliance.
\end{abstract}

Key words: Iron deficiency anemia, Iron polymaltose complex, haemoglobin, Serum iron, compliance.

\section{Introduction}

In India, which is a developing country, where population explosion is a major problem, the slogan of one or two children is very apt and therefore it is important to ensure that every fetus conceived is carried to viability and brought into this world under optimal conditions.

Anemia in pregnancy continues to play a major role in being responsible for maternal and fetal morbidity and mortality. The term 'Anemia in Pregnancy' refers to all types of anemia encountered during pregnancy. This term includes anemias occurring independently of pregnancy and also anemias precipitated or caused by pregnancy. Anemia is associated with increased preterm labor $(28.2 \%)$, preeclampsia $(31.2 \%)$, and maternal sepsis.[1] Severe anemia can lead to palpitations, tachycardia, breathlessness, and increased cardiac output leading to cardiac stress, which can cause decompensation and cardiac failure. Anemia is responsible for $40 \%-60 \%$ of maternal deaths in non-industrialized countries.[2]

Iron deficiency is the commonest cause of anemia all over the world. It is estimated that about $20 \%$ of women in child-bearing age group are iron deficient.

Pregnancy causes a state of hydremic plethora, in other words, the total volume of blood is increased partly by dilution and haemoglobin is consequently reduced to a varying extent, occasionally as low as $80 \%$.

Levels below this are pathological, and one should aim at raising the haemoglobin to $80 \%$ or more, if possible before delivery. The commonest source of trouble in anemic pregnancy is inadequate absorption of iron. The normal daily requirement for the gravid woman is about $20 \mathrm{mg}$ of iron, even in cases in which the iron stores have not suffered depletion prior to pregnancy, makes heavy demands upon maternal iron and average fetal requirements amount to about $375 \mathrm{mg}$. Unfortunately, the margin between the patients requirements and the quantity of iron normally available in a reasonably good diet is a very narrow one, infact, the average diet seldom contains more than about $15 \mathrm{mg}$ a day. Of the total amount of iron in food, only a fraction (about $10 \%$ ) is available for absorption. So, iron therapy is the sheet anchor in these women.[3]

However, patients do not always respond adequately to oral iron therapy because of noncompliance due to side effects. Gastrointestinal disturbances characterized by colicky pain, nausea, vomiting, diarrhea, and gastric distress occur in about $6 \%-12 \%$ of patients taking iron preparations. The most widely recommended oral iron is ferrous salts; however, the use of these salts is limited by low and variable absorption, chelation by food products, and free radical-mediated mucosal luminal damage.[4] Ferric compounds were introduced to avoid these problems. However, these compounds are generally less soluble at physiologic $\mathrm{pH}$ and precipitate 
intraluminally as hydroxide or phosphate and therefore have poor bioavailability. A need for a ferric complex that could overcome these problems was realized.[5]

Iron-polymaltose complex (IPC), a combination of ferric iron with maltol (a food additive), was developed as a molecule that is soluble at neutral $\mathrm{pH}$ and is not chelated by other substances.[6] Despite the advantage of the IPC over ferrous salts, the efficacy of IPC has not been well established in pregnancy. Studies have shown that IPC is as effective as Ferrous Sulphate (FS), or even more so.[7,8] But some studies contradict these results. $[9,10]$

Keeping the above view in mind, a prospective and comparative study was carried out in antenatal women to compare the efficacy, safety, compliance and cost effectiveness of IPC and FS given between 14 to 20 weeks of gestation.

\section{Materials And Methods}

The study was conducted in the Department of Pharmacology in collaboration with the Department of Obstetrics and Gynaecology at Government General Hospital, Guntur, Andhra Pradesh, India. A total of 120 patients were screened, and 100 were enrolled as 20 patients did not fulfill the inclusion criteria. Fifty patients were assigned to the FS group, and 50 patients were assigned to the IPC group.

In one group, iron polymaltose (Emcure limited, India), $100 \mathrm{mg}$ elemental iron + folic acid $500 \mathrm{mcg}$, was given for 6 weeks ( 1 tablet orally once daily). The other group received Ferrous Sulphate (GlaxoSmithKline, India)), $60 \mathrm{mg}$ elemental iron + folic acid $500 \mathrm{mcg}$, for 6 weeks (1 tablet orally twice daily). Folic acid was also given to both groups. Since FS tablets containing $50 \mathrm{mg}$ or $100 \mathrm{mg}$ of elemental iron are not available, the tablet containing $60 \mathrm{mg}$ of elemental iron was chosen and given twice daily. Patients were selected in accordance with the inclusion criteria:

- Pregnant;

- Age 20-40 years;

- Gestation 14-20 weeks;and

- $\mathrm{Hb} 6.5-8 \mathrm{~g} / \mathrm{dL}$ (moderate anemia).

Patients with a history of anemia due to any other causes such as chronic blood loss, hemolytic anemia, and thalassemia (including thalassemic trait) were excluded from the study. Pregnant women with $\mathrm{Hb}<6.5 \mathrm{~g} / \mathrm{dL}$ (severe anemia) were also excluded. Other exclusion criteria were as follows: clinical and/or laboratory evidence of hepatic, renal, hematologic, cardiovascular abnormalities; history of acid-peptic disorders, esophagitis, or hiatal hernia; family history of thalassemia, sickle cell anemia, or malabsorption syndrome; history of any other medical disorder; hypersensitivity to iron preparations; history of ingestion of hematinic within 24 hours prior to inclusion, and treatment with any other investigational drug in the last 1 month before study entry.

Written and informed consent was obtained from all patients in local language prior to screening and enrollment. The study protocol was approved by the Institutional ethics committee.

This was a 6-week, prospective, comparative, single-center study. The primary efficacy parameter was the proportion of women improving $\mathrm{Hb}$ level in the treatment groups within 6 weeks of treatment. Other efficacy parameters were packed cell volume (PCV), mean corpuscular haemoglobin concentration (MCHC) and serum iron. At Week 0 and Week 6, Hb, PCV, MCHC and Serum iron were measured. Hb and ADR were also measured in between the study at 2, 4, and 6 weeks.

\section{Investigations:}

1.1. Haemoglobin $(\mathrm{Hb})$ concentration was measured by Sahli's method.

Normal range: $12-14 \mathrm{~g} / \mathrm{dL}$

1.2. PCV was determined by micro-hematocrit method.[11]

Normal range: $37-42 \%$

1.3. $\mathrm{MCHC}$ was calculated by the formula:

$\mathrm{MCHC}(\mathrm{g} / \mathrm{L})=\mathrm{Hb}$ in $\mathrm{g} / \mathrm{dL} / \mathrm{PCV}$

Normal range: $30-36 \mathrm{~g} / \mathrm{dL}$

1.4. Serum iron was estimated by the International Committee for Standardisation in Haematology (ICSH) and based on the development of a coloured complex when ferrous iron is treated with a chromagen solution and the absorbance in a spectrophotometer against water at $562 \mathrm{~nm}$. Normal range: $60-180 \mu \mathrm{g} / \mathrm{dl} .[12]$

The patients were asked to report any unusual or unpleasant symptoms during the study period. This report included a detailed description of the signs and symptoms of the event, time of onset and duration, whether treatment was discontinued, corrective measures taken, outcome, and other possible causes. Compliance with study medication was determined by pill counting at each visit. 
Comparative study of efficacy and safety of Iron polymaltose complex with Ferrous sulphate in

\section{Statistical Analysis:}

Data were analyzed by using parametric tests. The differences between the groups for change from baseline to 6 weeks in haemoglobin, PCV, MCHC, serum iron and compliance (pill counting) were compared by using student's 2 -tailed unpaired ' $t$ ' test. The difference between the 2 groups with regard to primary efficacy parameter (percentage of patients improving $\mathrm{Hb}$ level within 6 weeks of treatment) and incidence of ADRs was compared by using chi-square $\left(\mathrm{x}^{2}\right)$ test. $P$ value $<.05$ was considered statistically significant.

\section{Results}

The study was initiated in January 2005 and completed in August 2005. All of the 100 patients enrolled in the study completed the entire 6 weeks. Data for all the 100 patients were included for analysis.

Baseline characteristics of all subjects and the baseline hematologic parameters including $\mathrm{Hb}$ concentration, $\mathrm{PCV}, \mathrm{MCHC}$, and serum iron were comparable between the 2 groups in table no. 1.

Table No. 1- Basic Parameters

\begin{tabular}{|l|l|c|c|}
\hline S.no. & \multicolumn{1}{|c|}{ Parameter } & FeSO4 & IPC \\
\hline 1. & No. of patients & 50 & 50 \\
\hline 2. & Median age years(range) & $22(18-24)$ & $20(19-24)$ \\
\hline 3. & Weight in kgs(mean \pm SD) & $47.2+5.23$ & $48.08+5.34$ \\
\hline 4. & Socioeconomic status & & 45 \\
& Low income group & 43 & 5 \\
\hline & Middle income group & 3 & 16 \\
\hline 5. & Food habits & & 34 \\
& Vegetarian & 23 & 21 \\
& Non-vegetarian & 27 & 20 \\
\hline 6. & Gravida & 20 & 6 \\
& Primi & 23 & 1 \\
\hline & Second & 5 & $7.253( \pm 0.46)$ \\
\hline 7. & Third & 1 & $30.04 \pm 1.948$ \\
\hline 8. & Fourth & $7.268( \pm 0.428)$ & $24.1 \pm 1.09$ \\
\hline 9. & MCV $\%($ mean \pm SD $)$ & $29.1 \pm 2.125$ & $71.24 \pm 11.28$ \\
\hline 10. & Serum iron mcg/dl(mean \pm SD) & $69.82 \pm 20.32$ & \\
\hline
\end{tabular}

Both groups showed a significant increase in $\mathrm{Hb}$ level (mean change from baseline was $1.05 \mathrm{~g} / \mathrm{dL}$ in FS group and $1.348 \mathrm{~g} / \mathrm{dL}$ in the IPC group which was a primary end point.

The data of the effect of ferrous sulphate and iron polymaltose complex were expressed as mean $\pm \mathrm{SD}$. Effects of FS and IPC on $\mathrm{Hb}(\mathrm{g} / \mathrm{L}), \mathrm{PCV}(\%), \operatorname{MCHC}(\mathrm{g} / \mathrm{L})$ and Serum Iron $(\mathrm{mcg} / \mathrm{dL})$ were tabulated in Table no. 2 .

Table no. 2: Data of effect of FS and IPC before and after treatment.

\begin{tabular}{|l|l|l|l|l|l|l|l|l|}
\hline $\begin{array}{l}\text { S. } \\
\text { No } \\
\end{array}$ & Parameter & \multicolumn{2}{|c|}{$\begin{array}{l}\text { Before treatment } \\
\text { IPC }\end{array}$} & \multicolumn{2}{|c|}{ FS After treatment } & \multicolumn{2}{|c|}{ Difference } & $\begin{array}{l}\text { Significa } \\
\text { nce } \\
\text { In P } \\
\text { value }\end{array}$ \\
\hline 1. & Hb & $7.268 \pm 0.428$ & $7.25 \pm 0.46$ & $8.318 \pm 0.392$ & $8.598 \pm 0.369$ & 1.05 & 1.348 & $<0.05$ \\
\hline 2. & PCV & $29.1 \pm 2.125$ & $30.04 \pm 1.948$ & $32.24 \pm 1.656$ & $34.24 \pm 1.656$ & 3.14 & 4.2 & $<0.05$ \\
\hline 3. & MCHC & $24.902 \pm 1.966$ & $24.1 \pm 1.09$ & $25.568 \pm 1.532$ & $25.09 \pm 1.120$ & 0.66 & 0.99 & $>0.05$ \\
\hline 4. & Sr. iron & $40.2 \pm 4.8$ & $44 \pm 4.4$ & $40.7 \pm 2.8$ & $47.7 \pm 2.7$ & 0.5 & 3.7 & $<0.001$ \\
\hline
\end{tabular}

The increase in PCV value was statistically significant when baseline vs 6-week values were compared in both groups. At the end of 6 weeks of either study treatment, there was a statistically significant increase in $\mathrm{MCHC}$ value from baseline in both treatment groups but there was no much difference between the 2 treatment groups.

Statistically significant increases in the serum iron levels were observed in both treatment groups. The baseline serum iron was $40.2 \pm 4.8 \mathrm{mcg} / \mathrm{dL}$ in FS group and $44 \pm 4.4 \mathrm{mcg} / \mathrm{dL}$ in the IPC group. In both groups, the serum iron level was increased statistically to $40.7 \pm 2.8 \mathrm{mcg} / \mathrm{dL}$ in the FS group and to $47.7 \pm 2.7 \mathrm{mcg} / \mathrm{dL}$ in the IPC group. When compared between the two groups the increase in serum iron was significantly different $(P<0.001)$. 


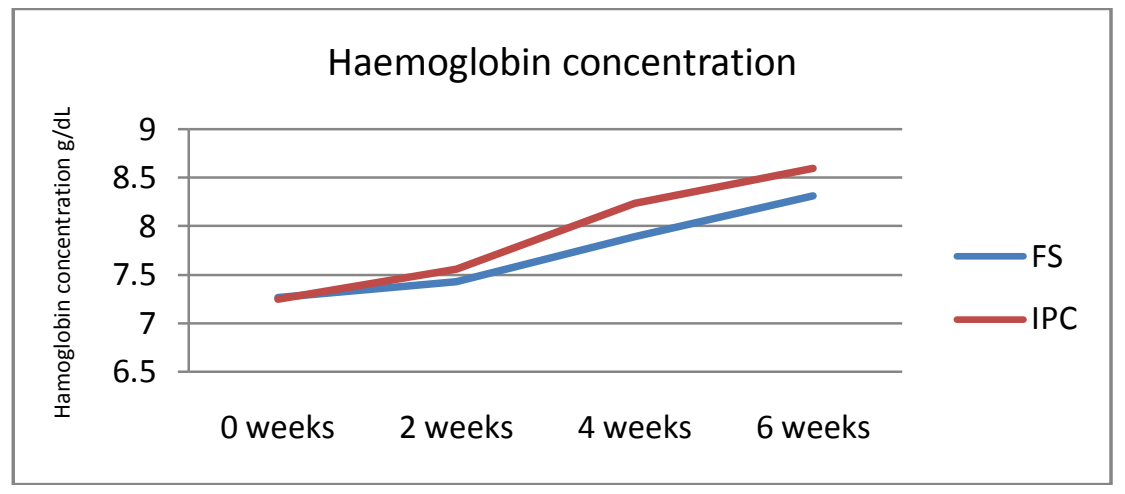

fig no.1: Mean $\mathrm{Hb}$ concentrations at $0,2,4$ and 6 weeks of treatment

Table no.3 Comparison of Adverse Events in FS and IPC Groups

\begin{tabular}{|l|l|l|l|}
\hline S.No & Adverse event & In FS group & In IPC group \\
\hline 1. & Nausea & 5 & 2 \\
\hline 2. & Vomiting & 2 & 0 \\
\hline 3. & Diarrhea & 6 & 1 \\
\hline 4. & Constipation & 2 & 6 \\
\hline 5. & Epigastric distress & 9 & 1 \\
\hline 6. & Metallic taste & 3 & 0 \\
\hline 7. & Rash & 0 & 0 \\
\hline
\end{tabular}

Table no. 3 shows the incidence of adverse effects experienced during the study. Overall, the adverse effects were more common in the FS group than in the IPC group [27 (51\%) in the FS group vs $10(20 \%)$ in the IPC group, $P<.001]$. The adverse effects experienced were nausea, vomiting, epigastric distress, constipation, metallic taste, diarrhea, and rash. The most common adverse effect was GI intolerance, seen in patients in both the FS and the IPC groups. Constipation was the second most common adverse effect seen with both of the drugs ( $48 \%$ in the FS group and $14 \%$ in the IPC group).

The compliance rate was higher than $80 \%$ in both groups. It was significantly higher $(P<.05)$ in the IPC group (91\%) than in the FS group (87\%).

The average drug cost was significantly higher for the IPC group (Rs $207.08 \pm 47.25)$ than for the FS group (Rs $139.98 \pm 75.51)(P<.001)$. The cost due to treatment of adverse effects was higher $(P<.001)$ in the FS group. So the average total cost (direct + indirect) of treatment of anemia for 6 weeks' duration in the FS group was Rs $1105.12 \pm 93.60$ and in the IPC group it was Rs $954.33 \pm 73.85$; this difference was not statistically significant. Cost (Direct + Indirect) of Iron Therapy in Ferrous Sulphate (FS) and Iron Polymaltose Complex (IPC) Groups (data expressed as Rs: $[$ mean $\pm \mathrm{SD}]$ )

\section{Discussion and Conclusion}

Anemia associated with pregnancy is a worldwide public health problem. A recent WHO report estimates the anemia prevalence among pregnant women to be $55.9 \%$ globally.[1] When it occurs during pregnancy, anemia has a significant impact on the health of the fetus as well as that of the mother.[2]

The treatment of IDA is directed at improving haemoglobin and compensating for the deficit in stored iron by supplying sufficient iron. In iron polymaltose complex, the elemental form is in a nonionic state. This causes less or no gastric irritation with iron polymaltose complex. In addition, the high elemental iron content of IPC eliminates the need for frequent dosing and therefore improves compliance.[6]

Other studies have shown similar results to those seen in the present study, but they had limitations. A study by Badhwar and colleagues[8] involving adult patients (both female and male) with IDA demonstrated comparable efficacy and superior bioavailability of IPC over ferrous fumarate. However, this study was conducted in nonpregnant women. A study by Patkar and colleagues also demonstrated efficacy and safety of IPC, in both pregnant and nonpregnant women, but this was an open, uncontrolled trial. [14] In another study, done by Rajadhyaksha, the efficacy and tolerability of IPC in IDA during pregnancy in Indian women were evaluated, and IPC was found to result in significantly reduced symptoms of anemia and significant improvements in serum iron, iron binding capacity, and $\mathrm{Hb}$; furthermore, only $8 \%$ of patients showed mild GIrelated adverse effects.[15] However, this study was limited by the absence of a control group. In the study undertaken by Reddy to evaluate the efficacy and safety of IPC in pregnant women with IDA, it was shown that the clinical parameters as well as biochemical parameters showed favorable changes with IPC.

In summary, none of these studies compared compliance and cost of iron preparations, and were conducted in nonpregnant women. 
In contrast to the aforementioned studies, in the present study compliance and cost were measured in both groups and compared, along with effectiveness and safety; these comparisons were not done in previous studies. However, our findings contradict those reported by Mehta; in a case report, he described the ineffectiveness of IPC in 4 pregnant patients with IDA.[9]

Coming to the adverse effects, they were more common in the FS group than in the IPC group in the present study. Similar observations were seen in the Indian populations (pregnant women) studied by Reddy and by Rajyadhyaksha. $[13,15]$

Increased incidence of adverse effects with FS may be due to release of free radicals, which leads to cell damage and cell death.[16] IPC does not release free radicals, which may account for the observed difference. Reduced incidence of adverse effects will improve patient compliance and ensure regular treatment. In the present study, the compliance rate was higher in the IPC group than in the FS group and the improvements seen in all hematologic parameters were comparable in both groups.

As drugs were provided to the patients by the investigators and the patients were directly observed by the investigators, the findings in this study cannot be extrapolated to the setting of general practice, where compliance rates may be lower and further reduced by the adverse effects seen with FS.

The pharmacoeconomic analysis shows that the 2 iron preparations are equally cost-effective. In the present study, the drugs (FS or IPC) were given to the patient at no cost, but in our analysis, the cost was calculated as per the retail price. Our institution, where the study was conducted, is a public sector government institute. So, the cost calculated in the present study cannot be extrapolated directly to private sector hospitals. Their costs may be higher than those seen in the present study.

In conclusion, IPC is effective in the treatment of iron deficiency anemia in pregnant women and unfortunately many pharmaceutical companies have stopped the production of it. A superior tolerability profile to that of the conventional iron preparation (FS) and an equivalent efficacy profile strongly suggest that it can be considered as a useful and alternative formulation for the treatment of IDA during pregnancy. However, further studies with large patient populations are required to confirm and strengthen the present study.

\section{Acknowledgements}

We are very thankful to Dr. C. Vasanth kumar, former Professor and head, department of Obstetrics and Gynaecology, for his cooperation and interest in providing facilities in the Obstetrics out-patient department.

We are also very thankful to Emcure ltd. for supplying drugs for the present study in time and for free of cost.

\section{References}

[1]. World Health Organization. The prevalence of anaemia in women: a tabulation of available information. Geneva, Switzerland: WHO; 1992.

[2]. Sharma JB. Nutritional anaemia during pregnancy in non-industrialised countries. In: Studd J, editor. Progress in Obstetrics and Gynaecology. New Delhi: Churchill Livingstone; 2003. pp. 103-122.

[3]. Robbins and Cotran, Pathologic basis of disease $8^{\text {th }}$ edition,(saunders Elsevier,2009) chapter-14, pg.no. 639-645.

[4]. Jacobs P, Johnson G, Wood L. Oral iron therapy in human subjects, comparative absorption between ferrous salts and iron polymaltose. J Med. 1984;15:367-377. [PubMed]

[5]. Tietz NW. In: Textbook of Clinical Chemistry. Tietz NW, editor. Philadelphia, Pa: WB Saunders Co.; 1986. pp. 1578-1583.

[6]. Jacobs P, Wormald LA, Gregory MC. Absorption of iron polymaltose and ferrous sulphate in rats and humans - a comparative study. S Afr Med J. 1979;26:1065-1072. [PubMed]

[7]. Reddy PSN, Adsul BB, Gandewar K, Korde KM, Desai A. Evaluation of efficacy and safety of iron polymaltose complex and folic acid (Mumfer) vs iron formulation (ferrous fumarate) in female patients with anemia. J Indian Med Assoc. 2001;99:154-155. [PubMed]

[8]. Badhwar VR, Rao S, Fonseca MM. Comparative efficacy and safety of iron polymaltose+folic acid and oral ferrous fumarate in the treatment of adult patients with iron deficiency anemia. Indian Med Gazette. 2003;136:296-301.

[9]. Mehta BC. Ineffectiveness of ironpolymaltose treatment of iron deficiency anaemia. Journal of the Association of Physicians of India. 2003;51:419-421. [PubMed]

[10]. Mehta BC. Iron hydroxide polymaltose complex -cause of persistent anemia at delivery. Indian J Med Sci. 2001;55:616-620. [PubMed]

[11]. England JM, Walford DM, Waters DAW. Re-assessment of the reliability of the haematocrit. Br J Haematol. 1972;23:247-256. [PubMed]

[12]. M.Worwood, Sir John V Dacie, S.M. Lewis, Practical Haematology, Iron deficiency anaemia, $8^{\text {th }}$ edn-pg 437-438.

[13]. Reddy PS, Adsul BB, Gandewar K, Desai A. Mumfer (iron polymaltose complex) in the management of anaemia in pregnancy - an Indian study. J Indian Med Assoc. 2000;98:343-346. [PubMed]

[14]. Patkar VD, Patkar S, Khandeparker PS, Dingankar NS, Shetty RS. Evaluation of efficacy and tolerability of iron (III) - hydroxide polymaltose complex tablets in the treatment of iron deficiency anaemia in women. Indian Med Gazette. 2001;135:306-309.

[15]. Rajadhyaksha GC, Shahani S, Pawar D. Evaluation of efficacy and tolerability of iron polymaltose complex tablets in iron deficiency anaemia during pregnancy. JAMA India. 2000;3:53-55

[16]. McCord JM. Iron, free radicals, and oxidative injury. Semin Hematol. 1998;35:5-12. [PubMed] 